\title{
Role of empathy in the perception of medical errors in patient encounters: a preliminary study
}

\author{
Jean Hannan ${ }^{*}$ (D), Gabriel Sanchez², Erica D. Musser ${ }^{3}$, Melissa Ward-Peterson ${ }^{4}$, Elizabeth Azutillo', \\ Deana Goldin ${ }^{5}$, Edgar Garcia Lara ${ }^{6}$, Aniuska M. Luna ${ }^{7}$, Igor Galynker ${ }^{8}$ and Adriana Foster ${ }^{9}$
}

\begin{abstract}
Objective: Healthcare professionals' empathy have been empirically demonstrated to decrease the risk of medical errors. Medical errors affect patient's outcomes and healthcare providers' well-being. Therefore, the purpose of this study was to determine the relationship between patients' perception of healthcare providers' empathy, their intention to adhere to treatment, and their perception of medical errors made. An anonymous survey was emailed to staff at a health center and an urban university in Miami, Florida, USA.

Results: A total of 181 participants were enrolled. Participants rating their healthcare provider as high in empathy had $80 \%$ lower odds of reporting errors (Cl 0.04-0.6). The intention to follow-up with recommendations or return to the provider were not significantly associated with provider's empathy. Patients of high empathy providers were no more treatment adherent that those who rated their provider with low empathy but were less likely to perceive medical error. Providers' empathy significantly affected patients' perception of medical errors. Our results underscore that healthcare curricula need to address the link between empathy and perception of medical errors, including its potential legal implications.
\end{abstract}

Keywords: Empathy, Medical error, Adherence, CARE measure, Communication

\section{Introduction}

Medical errors, defined as "failure of a planned action to be completed as intended, or the use of a wrong plan to achieve an aim," [1, 2] were estimated to contribute substantially to mortality in the United States [3]. Medical errors result in high individual and societal costs including lost quality of life, work productivity, and additional medical costs that amount to losses into the billions of dollars [4]. Yet medical errors are preventable. One way to reduce them is by enhancing perceived empathy in patient-healthcare provider interaction. Empathy in healthcare includes understanding the patient's

\footnotetext{
*Correspondence: jhann001@fiu.edu

${ }^{1}$ Nicole Wertheim College of Nursing and Health Sciences, Florida International University, 11200 SW 8th Street, Academic Health Center 3 Office 324A, Miami, FL 33199, USA

Full list of author information is available at the end of the article
}

perspective, communicating that understanding verbally and non-verbally, and acting therapeutically on that understanding [5]. Patients consider empathy to be very important in consultations, and show better treatment adherence and greater satisfaction with more empathetic doctors, while physicians' communication skills are associated with reduced risk of malpractice claims [5-7]. Levinson et al. [6] called the combination of a bad outcome and patient dissatisfaction, "a recipe for litigation". In face of a negative treatment result, a provider who relates to a patient in a "negative" manner (i.e. being perceived as less professional, caring, friendly, trustworthy) faces a higher risk of malpractice claims than a provider perceived to relate in a positive manner [8, 9]. Similarly, primary care providers who spend more time with the patient facilitate patient's involvement in their own care and use humor, face significantly less malpractice claims than providers who show poorer communication skills [6]. While healthcare training programs teach empathy 
early in their curricula [10-13], longitudinal reinforcement and assessment of this core communication skill varies. Furthermore, empathy, patients' perception of medical errors and prevention of litigation are addressed separately within the framework of healthcare professions' competencies $[14,15]$.

Considering these knowledge gaps and the potential benefits of enhancing patient-healthcare provider interactions through empathy, we investigated whether an association exists between patients' perception of healthcare providers' empathy, adherence to medical treatment and perception of medical errors. Our hypotheses were: (1) providers who demonstrate greater empathy elicit greater treatment adherence from their patients, and (2) patients perceive more empathetic providers as making less medical errors than providers who demonstrate lower empathy.

\section{Main text Methods}

An anonymous online survey created with Qualtrics software ${ }^{\circledR}$ was disseminated by email to a convenience sample of undergraduate and graduate Florida International University (FIU) nursing students, undergraduate FIU psychology students and FIU employees in Miami, Florida after IRB approval from FIU. The FIU IRB exempted the study waiving the need to obtain an informed consent by the participants being that data was collected anonymously using an online survey.

The same survey was disseminated to healthcare professionals, staff and trainees (psychiatry and psychology residents and psychology postdoctoral students) at a Federally Qualified Health Center, Citrus Health Network in Hialeah, Florida. The study took place in March and April 2018. FIU Institutional Review Board approved the study.

To elicit the patients' perception of providers' empathy, we utilized the Consultation and Relational Empathy (CARE) measure [16]. CARE is a validated 10-items instrument that measures patients' perception of physician's empathy in the medical encounter. Each item is measured on a 5-point scale (Poor, Fair, Good, Very Good and Excellent). CARE minimum score is 10 and maximum is 50. Normative data on CARE measure identify high-empathy (CARE score 10-30), middle-empathy (score 31-40) and low-empathy providers (score 41-50) $[17,18]$. Lower CARE scores of primary care physicians have been associated with poorer patient outcomes [17, 18] and have improved after educational interventions targeting empathy [19].

The CARE measure was preceded by a prompt asking the participants to recall their last health care encounter and questions on whether they had followed through with advice would return to that provider, and if they thought, the provider made any medical errors. A definition of medical error (Kohen [1] "a failure in the process of care that could have been prevented") was provided in the prompt. The CARE measure was followed by a section requesting demographic background, income bracket and health insurance coverage status. A final prompt allowed participants to submit comments about their experiences ("If you would like to add further comments on this consultation, please do so here").

We examined descriptive statistics (frequency distributions for categorical variables, mean and standard deviation for continuous variables) for demographics and the outcome variables of interest (follow through with recommendations, intention to return and perception of medical errors). Based on the empathy rating of their last healthcare provider encounter using the CARE measure, survey participants were placed into high, middle and low empathy groups [18]. In order to identify potential covariates, bivariate analyses (using either Chi square tests or independent two sample t-tests) were carried out to examine associations between provider empathy group, survey participant demographics and the outcome variables. After identifying covariates, we used multivariable binary logistic regression models to estimate odds ratios with $95 \%$ confidence intervals. We estimated separately the odds the survey participants': (1) follow-through with provider's recommendations, (2) intention to return and (3) perception of a medical error. The empathy score group was the main exposure of interest in all models, with the low-empathy provider group considered the reference group. We used Stata 14 software for all analysis.

\section{Results}

Of 195 survey participants, only 181 had complete data on CARE empathy score, gender, and income and thus were included in the final models. Demographic information is summarized in Table 1. Final adjusted models controlled for gender and income.

The mean CARE survey score among our participants was 38.94 (SD 9.73), as illustrated in Table 2. To establish whether the low, middle and high empathy scores correlated with perceived error and patient adherence by survey participants, we performed logistic regression analysis and calculated odds ratios. Participants who rated their provider as having high empathy, as compared to those who rated them as having low empathy, had $80 \%$ lower odds of reporting medical error (CI 0.04-0.6) and had $220 \%$ higher odds of follow through with providers recommendations (CI 0.8-5.8). The latter finding was not statistically significant at a $95 \%$ confidence interval, but it is noteworthy because it approaches significance. Those who perceived their physician as showing "middle" empathy score showed no statistically significant difference 
Table 1 Demographics $(n=181)$

\begin{tabular}{|c|c|c|}
\hline & Frequency & Percent \\
\hline \multicolumn{3}{|l|}{ Gender } \\
\hline Male & 40 & 22.10 \\
\hline Female & 141 & 77.90 \\
\hline \multicolumn{3}{|l|}{ Race } \\
\hline White & 127 & 70.17 \\
\hline Black & 17 & 9.39 \\
\hline Other & 28 & 15.47 \\
\hline Unknown & 9 & 4.97 \\
\hline \multicolumn{3}{|l|}{ Ethnicity } \\
\hline Hispanic/Latino & 102 & 56.35 \\
\hline Other & 69 & 38.12 \\
\hline Unknown & 10 & 5.52 \\
\hline \multicolumn{3}{|l|}{ Income (USD) } \\
\hline $0-49,400$ & 56 & 30.94 \\
\hline $49,401-127,550$ & 85 & 46.96 \\
\hline$>127,550$ & 40 & 22.10 \\
\hline \multicolumn{3}{|l|}{ Insurance } \\
\hline Private Health Insurance & 165 & 91.16 \\
\hline Medicaid/Medicare & 8 & 4.42 \\
\hline VA Care & 1 & 0.55 \\
\hline Uninsured & 6 & 3.31 \\
\hline \multirow[t]{2}{*}{ Other } & 1 & 0.55 \\
\hline & Mean & SD \\
\hline $\mathrm{Age}^{\mathrm{a}}$ & 33.51 & 12.12 \\
\hline
\end{tabular}

a Age only available for 175 of 181 respondents

Table 2 Distribution of patients' perception of healthcare providers' empathy

\begin{tabular}{lcc}
\hline & $\mathbf{N}=\mathbf{1 8 1}$ subjects & Percentage \\
\hline CARE score categories & 43 & \\
Low & 43 & 23.76 \\
Middle & 95 & 24.76 \\
High & & 52.49 \\
Follow recommendations & 31 & \\
No & 150 & 17.13 \\
Yes & & 82.87 \\
Return to provider & 14 & 7.73 \\
No & 167 & 92.27 \\
Yes & & \\
Provider made error & 166 & 91.71 \\
No & 15 & 8.29 \\
Yes & & \\
\hline
\end{tabular}

in odds of either following through with providers' recommendations or reporting medical error compared to those who rated their provider as having low empathy (Table 3). Regarding likelihood to return to provider, very few participants rated empathy as medium or high and said they would not return to the provider (Table 2).

\section{Discussion}

The mean CARE survey score among our participants was 38.94 with a standard deviation of 9.73, consistent with a meta-analysis of CARE survey results, which found a mean of 40.48 [20]. Our data indicates that patients who perceived their healthcare providers as having high empathy, as compared to those who rated them as having low empathy, had significantly lower odds of reporting medical error.

Patient dis/satisfaction, willingness to follow medical advice and compliance, and perception of empathy were reflected in the scarce descriptive statements provided at the end of the survey. For instance, a respondent who stated: "I recently changed physicians and I can honestly say that this physician surprised me. He truly took the time to listen to my concerns amidst the other patients he had waiting. I did not feel like I was simply another patient, but a unique individual. I believe that this is a rare quality to find in physicians since they tend to be overwhelmed with work", also answered that s/he would go back to said physician, would follow up on his recommendations, and perceived him to not have committed medical errors. The remaining answers in the participant's survey rated all aspects of their interaction as "excellent".

Approach-based limitations of the study included the one-time, cross-sectional assessment of the relationship between the survey participants and their healthcare providers. In addition, we did not elicit information about the type of healthcare providers rated by our survey participants, nor did we elicit the duration since the participants' last healthcare encounter. Our patient sample was small, contained mostly white, female, Hispanic participants and the inquiry was retrospective. Of those who rated their providers' empathy as medium or high very few said they would not return to the provider, thus leading to wide confidence intervals. Finally, although they offered a few comments, the survey participants did not provide enough details to help characterize medical errors and experiences of empathy linked to their encounters in a significant manner.

\section{Conclusion}

On our relatively small sample of survey participants representing a diverse population in a densely populated urban area, physicians' empathy significantly affected patients' perception of medical errors, validating prior work $[6,8,9]$ but it did not affect treatment adherence. These results must be replicated in larger studies. The 
Table 3 Association between physicians' empathy, patient adherence and patient perception of medical errors

\begin{tabular}{|c|c|c|c|}
\hline & $\begin{array}{l}\text { Follow recommendations } \\
\text { Adjusted OR }(95 \% \mathrm{Cl})^{\mathrm{a}}\end{array}$ & Return to provider & $\begin{array}{l}\text { Errors in care } \\
\text { Adjusted OR }(95 \% \mathrm{Cl})\end{array}$ \\
\hline \multicolumn{4}{|l|}{ CARE score ${ }^{b}$} \\
\hline Low (10-30) & Used as reference & & \\
\hline Middle (31-40) & $1.5(0.5-4.3)$ & $7.0(1.3-37.5)$ & $0.5(0.1-1.8)$ \\
\hline High (41-50) & $2.2(0.8-5.8)$ & $15.3(2.9-80.7)$ & $0.2(0.04-0.6)$ \\
\hline
\end{tabular}

Empathy categories defined by Steinhausen et al. [18]

OR odds ratio, $\mathrm{Cl}$ confidence interval (95\%)

a Adjusted models controlled for gender and income

b CARE

results underscore not only the need of teaching and reinforcing empathy throughout healthcare professions' curricula, but also the immediate need to focus training on the link between empathy, perception of medical errors and their potential legal and quality of care implications.

\section{Practice implications}

In this study physicians' empathy significantly affected patients' perception of medical errors, suggesting that empathy may be inversely related to the potential legal implications of un-empathic care (i.e. malpractice lawsuits). Therefore, teaching empathy as part of medical errors prevention programs throughout healthcare professions curricula and post-licensure may improve quality of life for patients and healthcare providers and prevent the personal, social and economic burden associated with errors.

\section{Limitations}

- A relatively small sample.

- Limited to one area geographically.

\begin{abstract}
Abbreviations
CARE: Consultation and Relational Empathy; FIU: Florida International University.
\end{abstract}

\section{Acknowledgements}

The authors are grateful to the people who took the time to fill out our survey, and to the FIU administration and NWCHN for facilitating the dissemination of our survey. Melissa Ward-Peterson is currently supported by an NIMHD center grant to the Research Center in Minority Institutions at Florida International University (U54MD012393)

\section{Authors' contributions}

Each author listed (JH, GS, EM, MWP, EA, DG, EG, AL, IG, AF) made substantial contributions to the conception, design of the work; the acquisition, analysis, interpretation of data; and drafted the work and substantively revised it. All authors (JH, GS, EM, MWP, EA, DG, EG, AL, IG, AF) approved the submitted version. All authors (JH, GS, EM, MWP, EA, DG, EG, AL, IG, AF) agreed to be personally accountable for the author's own contributions and to ensure questions related to the accuracy or integrity of any part of the work, even ones in which the author was not personally involved, are appropriately investigated, resolved, and the resolution documented in the literature. All authors read and approved the final manuscript.

\section{Funding}

This research did not receive any specific grant from funding agencies in the public, commercial, or not-for-profit sectors.

\section{Availability of data and materials}

All data generated or analyzed during this study are included in this published article.

\section{Ethics approval and consent to participate}

IRB was obtained by Florida Internationals Internal Review board. The FIU IRB exempted the study waiving the need to obtain an informed consent by the participants being that data was collected anonymously using an online survey.

\section{Consent for publication}

Not applicable.

\section{Competing interests}

The authors declare that they have no competing interests.

\section{Author details \\ ${ }^{1}$ Nicole Wertheim College of Nursing and Health Sciences, Florida Interna- tional University, 11200 SW 8th Street, Academic Health Center 3 Office 324A, Miami, FL 33199, USA. ${ }^{2}$ Citrus Health Network/Florida International University, 4175 West 20th Ave, Hialeah, FL 33012, USA. ${ }^{3}$ Center for Children and Families, 11200 SW 8th Street, AHC 4, Room 455, Miami, FL 33199, USA. ${ }^{4}$ Department of Epidemiology, Florida International University, Robert Stempel College of Public Health \& Social Work, 11200 SW 8th Street, AHC5-483, Miami, FL 33199, USA. ${ }^{5}$ Nicole Wertheim College of Nursing and Health Sciences, Florida International University, 11200 SW 8th Street, AHC 3 Office 228, Miami, FL 33199, USA. ${ }^{6}$ Miami Dade College Benjamin Leon School of Nursing, 950 NW 20th Street, Miami, FL 33127, USA. ${ }^{7}$ Department of Psychiatry \& Behavioral Health, Herbert Wertheim College of Medicine, Florida International Univer- sity, 11200 SW 8th Street, AHC4 280, Miami, FL 33199, USA. ${ }^{8}$ Icahn School of Medicine, Beth Israel-Fierman, 317 E 17th St, New York, NY 10003, USA. \\ ${ }^{9}$ Department of Psychiatry \& Behavioral Health, Herbert Wertheim College of Medicine, Florida International University, 11200 SW 8th Street, AHC1 335A, Miami, FL 33199, USA.}

Received: 14 March 2019 Accepted: 5 June 2019

Published online: 10 June 2019 


\section{References}

1. Kohn LT, Corrigan JM, Donaldson MS. To err is human: building a safer health system. Washington, DC: Institute of Medicine (US) Committee on Quality of Health Care in America; National Academies Press (US); 2000. https://doi.org/10.17226/9728.

2. Graber ML, Franklin N, Gordon R. Diagnostic error in internal medicine. Arch Intern Med. 2005;165(13):1493-9.

3. Makary MA, Daniel M. Medical error-the third leading cause of death in the US. BMJ. 2016. https://doi.org/10.1136/bmj.i2139.

4. Andel C, Davidow SL, Hollander M, Moreno DA. The economics of health care quality and medical errors. J Health Care Finance. 2012;39(1):39-50.

5. Mercer SW, Reynolds WJ. Empathy and quality of care. Br J Gen Pract. 2002;52(Suppl):S9-12.

6. Levinson W, Roter DL, Mullooly JP, Dull VT, Frankel RM. Physician-patient communication: the relationship with malpractice claims among primary care physicians and surgeons. JAMA. 1997;277(7):553-9.

7. Wensing M, Jung HP, Mainz J, Olesen F, Grol R. A systematic review of the literature on patient priorities for general practice care. Part 1: description of the research domain. Soc Sci Med. 1998;47(10):1573-88.

8. Shapiro RS, Simpson DE, Lawrence SL, Talsky AM, Sobocinski KA Schiedermayer DL. A survey of sued and nonsued physicians and suing patients. Arch Intern Med. 1989;149(10):2190-6.

9. Lester GW, Smith SG. Listening and talking to patients. A remedy for malpractice suits? West J Med. 1993;158(3):268-72

10. Hojat M. Ten approaches for enhancing empathy in health and human services cultures. J Health Hum Serv Adm. 2009;31(4):412-50.

11. Brunero S, Lamont S, Coates M. A review of empathy education in nursing. Nurs Inq. 2010;17(1):65-74. https://doi.org/10.111 1/j.1440-1800.2009.00482.x.

12. Potter PA, Perry AG, Stockert P, Hall A. Fundamentals of nursing, chapter 24. 8th ed. Amsterdam: Elsevier Health Sciences; 2013.

13. Batt-Rawden S, Chisolm MS, Anton B, Flickinger TE. Teaching empathy to medical students: an updated, systematic review. Acad Med. 2013;88(8):1171-7. https://doi.org/10.1097/ACM.0b013e318299f3e3.
14. Quality and Safety Education for Nurses (QSEN). 2019. http://qsen.org/ competencies/pre-licensure-ksas/. Accessed 10 Jan 2019.

15. ACGME Clinical Learning Environment (CLER). 2017. http://www.acgme .org/Portals/0/PDFs/CLER/CLER_Pathways_V1.1_Digital_Final.pdf. Accessed 17 June 2018

16. Mercer SW, Maxwell M, Heaney D, Watt GC. The consultation and relational empathy (CARE) measure: development and preliminary validation and reliability of an empathy-based consultation process measure. Fam Pract. 2004:21(6):699-705.

17. Mercer SW, Higgins M, Bikker AM, Fitzpatrick B, McConnachie A, Lloyd SM, Watt GC. General practitioners' empathy and health outcomes: a prospective observational study of consultations in areas of high and low deprivation. Ann Fam Med. 2016;14(2):117-24. https://doi.org/10.1370/ afm.1910.

18. Steinhausen S, Ommen O, Antoine SL, Koehler T, Pfaff H, Neugebauer E. Short- and long-term subjective medical treatment outcome of trauma surgery patients: the importance of physician empathy. Patient Prefer Adherence. 2014;8:1239-53. https://doi.org/10.2147/ppa.s62925.

19. Riess H, Kelley JM, Baile RW, Dunn EJ, Phillips M. Empathy training for resident physicians: a randomized controlled trial of a neuroscienceinformed curriculum. J Gen Intern Med. 2012;27(10):1280-6.

20. Howick J, Steinkopf L, Ulyte A, Roberts N, Meissner K. How empathic is your healthcare practitioner? A systematic review and meta-analysis of patient surveys. BMC Med Educ. 2017;17:136. https://doi.org/10.1186/ s12909-017-09.

\section{Publisher's Note}

Springer Nature remains neutral with regard to jurisdictional claims in published maps and institutional affiliations.
Ready to submit your research? Choose BMC and benefit from:

- fast, convenient online submission

- thorough peer review by experienced researchers in your field

- rapid publication on acceptance

- support for research data, including large and complex data types

- gold Open Access which fosters wider collaboration and increased citations

- maximum visibility for your research: over $100 \mathrm{M}$ website views per year

At BMC, research is always in progress.

Learn more biomedcentral.com/submissions 